\title{
Chapter 39 \\ Report on Global Environment \\ Competitiveness of Congo, Rep.
}

Congo is a country located in Central Africa. It is bordered by Gabon, Cameroon, the Central African Republic, the Democratic Republic of the Congo, and the Angolan exclave of Cabaña.It covers 341.5 thousand of square kilometers and has a population of 4.14 million. Its GDP reaches $\$ 14.43$ billion in 2011 . Through the index system

\begin{tabular}{|l|}
\hline Score: \\
50.92 \\
\hline Rank: \\
$\mathbf{5 7}$ \\
\hline
\end{tabular}
and evaluation model, the environment competitiveness index of Congo, Rep. ranks at 57 in 133 countries.

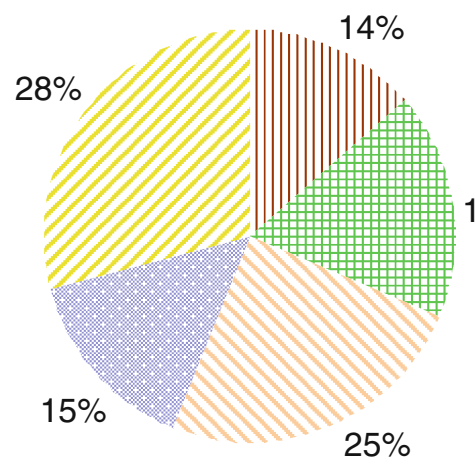

II Resource Environment Competitiveness

* Ecological Environment Competitiveness

Environment Carrying Competitiveness

* Environment Management Competitiveness

Fig. 39.1 Contribution Environment Harmony of sub-index to GEC Competitiveness 


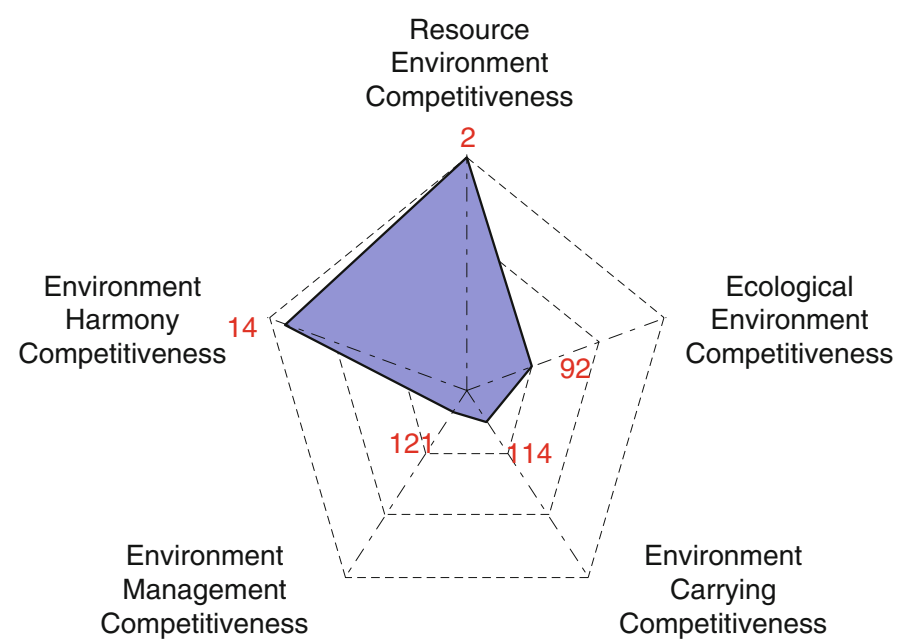

Fig. 39.2 Rank of sub-index of GEC

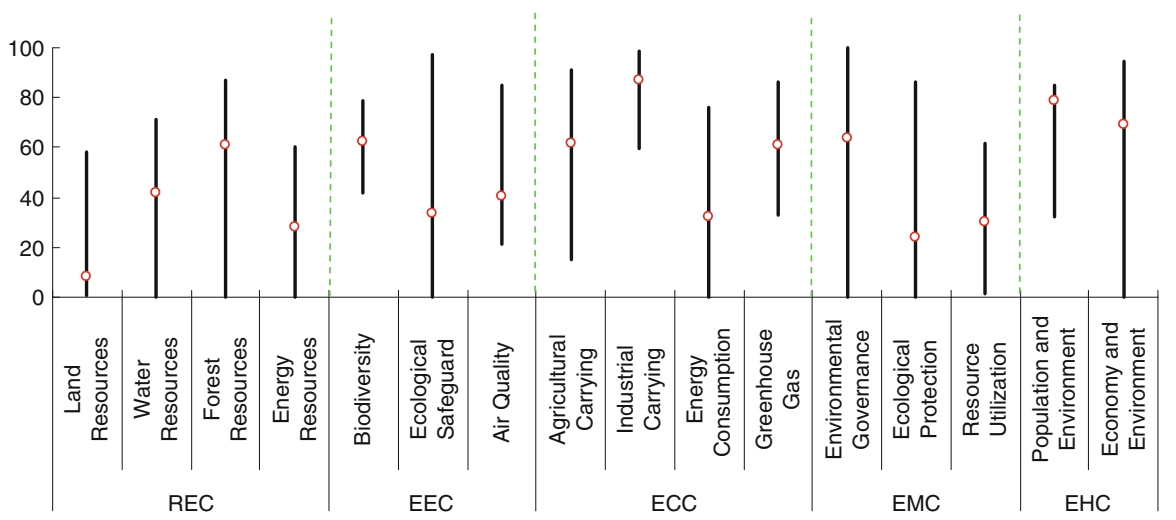

Fig. 39.3 Score and rank of the pillars of GEC

Table 39.1 Score and rank of all indicators of GEC

\begin{tabular}{|c|c|c|c|c|c|}
\hline Indicators & Score & Rank & Indicators & Score & Rank \\
\hline 1 Resource Environment & 34.79 & 2 & Groundwater & 51.44 & 9 \\
\hline Competitiveness & & & \multirow{2}{*}{$\begin{array}{l}\text { Total internal renewable } \\
\text { water resources }\end{array}$} & \multirow[t]{2}{*}{32.79} & \multirow[t]{2}{*}{36} \\
\hline 1.1 Land Resources & 8.33 & 95 & & & \\
\hline Land area per capita & 14.85 & 14 & 1.3 Forest Resources & 61.17 & 5 \\
\hline $\begin{array}{l}\text { Percentage of arable land } \\
\text { to total land area }\end{array}$ & 2.31 & 121 & $\begin{array}{l}\text { Growing stock in forest } \\
\text { and other wooded land }\end{array}$ & 63.75 & 10 \\
\hline Arable land per capita & 5.65 & 89 & \multirow{2}{*}{$\begin{array}{l}\text { Proportion of land area } \\
\text { covered by forest }\end{array}$} & \multirow[t]{2}{*}{76.82} & \multirow[t]{2}{*}{6} \\
\hline 1.2 Water Resources & 41.60 & 20 & & & \\
\hline Surface water & 26.30 & 5 & Forest area per capita & 37.73 & 7 \\
\hline Annual precipitation & 55.89 & 26 & 1.4 Energy Resources & 28.03 & 5 \\
\hline
\end{tabular}


Table 39.1 (continued)

\begin{tabular}{|c|c|c|c|c|c|}
\hline Indicators & Score & Rank & Indicators & Score & Rank \\
\hline Fossil energy & 1.45 & 29 & \multirow{2}{*}{$\begin{array}{l}\text { Energy consumption per unit } \\
\text { of land area }\end{array}$} & \multirow[t]{2}{*}{99.99} & \multirow[t]{2}{*}{4} \\
\hline Energy production & 4.33 & 21 & & & \\
\hline \multirow{2}{*}{$\begin{array}{l}\text { Proportion of combustible } \\
\text { renewable and waste to } \\
\text { total energy consumption }\end{array}$} & \multirow[t]{2}{*}{55.40} & \multirow[t]{2}{*}{22} & $\begin{array}{l}\text { Ratio of clean energy } \\
\text { consumption }\end{array}$ & 3.45 & 77 \\
\hline & & & Elasticity of energy & 13.98 & 60 \\
\hline $\begin{array}{l}\text { Net energy imports of the } \\
\text { energy consumption }\end{array}$ & 100.00 & 1 & \multirow{2}{*}{$\begin{array}{l}\text { Elasticity of electric power } \\
\text { consumption }\end{array}$} & \multirow[t]{2}{*}{11.86} & \multirow[t]{2}{*}{33} \\
\hline \multirow{2}{*}{$\begin{array}{l}2 \text { Ecological Environment } \\
\text { Competitiveness }\end{array}$} & \multirow[t]{2}{*}{44.81} & \multirow[t]{2}{*}{92} & & & \\
\hline & & & 3.4 Greenhouse Gas & 60.74 & 75 \\
\hline 2.1 Biodiversity & 61.99 & 12 & Growth rate of $\mathrm{CO}_{2}$ & 39.19 & 112 \\
\hline Threatened fish species & 78.30 & 102 & emissions & & \\
\hline Threatened mammal species & 94.02 & 64 & Growth rate of Methane & 53.28 & 98 \\
\hline Threatened plant species & 97.84 & 92 & emissions & & \\
\hline $\begin{array}{l}\text { GEF benefits index for } \\
\text { biodiversity }\end{array}$ & 19.90 & 19 & $\begin{array}{l}\mathrm{CO}_{2} \text { emissions per unit of } \\
\text { land area }\end{array}$ & 100.00 & 8 \\
\hline 2.2 Ecological Safeguard & 33.51 & 35 & $\mathrm{CO}_{2}$ emissions per unit of & 72.02 & 21 \\
\hline Terrestrial protected areas & 26.90 & 70 & energy consumption & & \\
\hline Marine protected areas & 43.43 & 5 & 4 Environment Management & 37.76 & 121 \\
\hline 2.3 Air Quality & 40.40 & 125 & eness & & \\
\hline Inhalable particles (PM10) & 58.39 & 106 & 4.1 Environmental & 63.50 & 115 \\
\hline Particulate matter (PM2.5) & 58.02 & 125 & & $N / A$ & $\mathrm{~N} / \mathrm{A}$ \\
\hline Index of indoor air pollution & 2.50 & 115 & $\begin{array}{l}\text { Agricuinural cnemicals } \\
\text { regulation }\end{array}$ & N/A & N/A \\
\hline Nitrogen oxides emission & 68.25 & 51 & Percentage of the rural & 32.00 & 120 \\
\hline Sulfur dioxide emission & 40.83 & 46 & population with access & & \\
\hline $\begin{array}{l}3 \text { Environment Carrying } \\
\text { Competitiveness }\end{array}$ & 63.30 & 114 & $\begin{array}{l}\text { to an improved water } \\
\text { source }\end{array}$ & & \\
\hline 3.1 Agricultural Carrying & 61.84 & 105 & Percentage of the urban & 95.00 & 93 \\
\hline $\begin{array}{l}\text { Cereal yield per unit of } \\
\text { arable land }\end{array}$ & 4.66 & 124 & $\begin{array}{l}\text { population with access } \\
\text { to an improved water }\end{array}$ & & \\
\hline Fertilizer consumption per & 99.92 & 5 & source & & \\
\hline un & & & 4.2 Ecological Protection & 24.15 & 95 \\
\hline $\begin{array}{l}\text { Annual freshwater } \\
\text { withdrawals for }\end{array}$ & 99.99 & 3 & $\begin{array}{l}\text { Area of plantation and } \\
\text { afforestation }\end{array}$ & 0.08 & 92 \\
\hline agriculture per unit of & & & Biome protect & 55.40 & 68 \\
\hline $\begin{array}{l}\text { arable land } \\
\text { 3.2 Industrial Carrying }\end{array}$ & 87.12 & 101 & $\begin{array}{l}\text { Overfishing of fishing } \\
\text { resources }\end{array}$ & 25.00 & 93 \\
\hline Net exports of goods as a & 48.73 & 128 & 4.3 Resource Utilization & 30.16 & 97 \\
\hline $\begin{array}{l}\text { percentage of GDP } \\
\text { Electric power consumption }\end{array}$ & 100.00 & 1 & $\begin{array}{l}\text { Utilization rate of water } \\
\text { resources }\end{array}$ & 0.02 & 123 \\
\hline $\begin{array}{l}\text { per unit of value added } \\
\text { of industry }\end{array}$ & & & $\begin{array}{l}\text { Percentage of total internal } \\
\text { renewable water }\end{array}$ & 23.27 & 112 \\
\hline $\begin{array}{l}\mathrm{SO}_{2} \text { emissions per unit of } \\
\text { value added of industry }\end{array}$ & 99.84 & 62 & $\begin{array}{l}\text { resources to total water } \\
\text { resources }\end{array}$ & & \\
\hline $\begin{array}{l}\text { Annual freshwater } \\
\text { withdrawals for industry }\end{array}$ & 99.90 & 9 & $\begin{array}{l}\text { Percentage of agricultural } \\
\text { land to total land area }\end{array}$ & 36.55 & 96 \\
\hline $\begin{array}{l}\text { per value added of } \\
\text { industry }\end{array}$ & & & $\begin{array}{l}\text { Percentage of fossil fuel } \\
\text { energy consumption to }\end{array}$ & 60.79 & 23 \\
\hline 3.3 Energy Consumption & 32.32 & 75 & total energy consumption & & \\
\hline
\end{tabular}


Table 39.1 (continued)

\begin{tabular}{lcclcc}
\hline Indicators & Score & Rank & Indicators & Score & Rank \\
\hline $\begin{array}{l}5 \text { Environment Harmony } \\
\text { Competitiveness }\end{array}$ & 73.95 & 14 & $\begin{array}{l}\mathrm{CO}_{2} \text { emissions per capita } \\
\text { Energy consumption } \\
\text { per capita }\end{array}$ & 99.00 & 23 \\
$\begin{array}{c}5.1 \text { Population and } \\
\text { Environment }\end{array}$ & 78.85 & 22 & $\begin{array}{l}\text { 5.2 Economy and } \\
\text { Environment }\end{array}$ & 69.05 & 36 \\
$\begin{array}{c}\text { Percentage of population } \\
\text { with access to Improved } \\
\text { sanitation facilities }\end{array}$ & 30.00 & 114 & $\begin{array}{c}\text { Land resource utilization } \\
\text { efficiency }\end{array}$ & 0.01 & 115 \\
$\begin{array}{c}\text { Motor vehicles per 1,000 } \\
\text { people }\end{array}$ & 97.04 & 26 & $\begin{array}{c}\text { Sulfur dioxide emissions per } \\
\text { unit of GDP }\end{array}$ & 85.08 & 104 \\
$\begin{array}{c}\text { Renewable internal } \\
\text { freshwater resources } \\
\text { per capita }\end{array}$ & 64.89 & 6 & $\begin{array}{c}\text { Carbon dioxide emissions } \\
\text { per unit of GDP }\end{array}$ & 97.12 & 6 \\
$\mathrm{SO}_{2}$ emissions per capita & 94.16 & 78 & $\begin{array}{c}\text { Energy consumption per unit } \\
\text { of GDP }\end{array}$ & 93.98 & 22 \\
\hline
\end{tabular}

Table 39.2 Rank distribution of the individual indicators of GEC

\begin{tabular}{lllllll}
\hline & $\begin{array}{l}\text { Number of } \\
\text { the individual } \\
\text { indicators }\end{array}$ & $\begin{array}{l}\text { Rank } \\
1-10\end{array}$ & $\begin{array}{l}\text { Rank } \\
11-30\end{array}$ & $\begin{array}{l}\text { Rank } \\
31-60\end{array}$ & $\begin{array}{l}\text { Rank } \\
61-100\end{array}$ & $\begin{array}{l}\text { Rank } \\
101-133\end{array}$ \\
\hline $\begin{array}{c}\text { Sub-index } \\
\text { Resource Environment } \\
\quad \text { Competitiveness } \\
\begin{array}{c}\text { Ecological Environment } \\
\quad \text { Competitiveness }\end{array}\end{array}$ & 14 & 6 & 6 & 0 & 1 & 1 \\
$\begin{array}{c}\text { Environment Carrying } \\
\quad \text { Competitiveness }\end{array}$ & 15 & 0 & 1 & 2 & 3 & 5 \\
$\begin{array}{c}\text { Environment Management } \\
\quad \text { Competitiveness }\end{array}$ & 10 & 4 & 0 & 1 & 5 & 5 \\
$\begin{array}{c}\text { Environment Harmony } \\
\text { Competitiveness }\end{array}$ & 10 & 0 & 0 & 0 & 5 & 4 \\
Total & 60 & 2 & 3 & 1 & 1 & 3 \\
\hline
\end{tabular}

Open Access This chapter is distributed under the terms of the Creative Commons Attribution Noncommercial License, which permits any noncommercial use, distribution, and reproduction in any medium, provided the original author(s) and source are credited. 